\title{
The shape of incident shock wave in steady axisymmetric conical Mach reflection
}

\author{
Yu-xin $\operatorname{Ren}^{1 *}$ (D), Lianhua $\operatorname{Tan}^{2}$ and Zi-niu Wu ${ }^{1}$
}

\author{
* Correspondence: ryx@tsinghua. \\ edu.cn \\ ${ }^{1}$ School of Aerospace Engineering, \\ Tsinghua University, Beijing 100084, \\ China \\ Full list of author information is \\ available at the end of the article
}

\begin{abstract}
For internal flow with supersonic inflow boundary conditions, a complicated oblique shock reflection may occur. Different from the planar shock reflection problem, where the shape of the incident shock can be a straight line, the shape of the incident shock wave in the inward-facing axisymmetric shock reflection in steady flow is an unknown curve. In this paper, a simple theoretical approach is proposed to determine the shape of this incident shock wave. The present theory is based on the steady Euler equations. When the assumption that the streamlines are straight lines at locations just behind the incident shock is adopted, an ordinary differential equation can be derived, and the shape of the incident shock wave is given by the solution of this ordinary differential equation. The predicted curves of the incident shock wave at several inlet conditions agree very well with the results of the numerical simulations.
\end{abstract}

Keywords: Axisymmetric shock reflection, The shape of incident shock wave, Euler equations

\section{Introduction}

Understanding the characteristics of the shock waves is important in the design of supersonic vehicles. Li and Ben-Dor [1] used several examples to show the great influence of the shock waves on the operating conditions of the inlet/combustor of a hypersonic craft, on the heating loads of a blunt body, and on the initiation of the detonation in a ram accelerator. In the axisymmetric supersonic internal flows, an oblique inward-facing conical shock will steepen near the symmetry axis, which has been observed by Mölder et al. [2]. As a result, transition to Mach reflection has to occur, so that regular reflection is not possible (Hornung and Schwendeman [3]). In contrast to the planar shock reflection problem where the shape of the incident shock can be a straight line, the shape of the incident shock wave in the inward-facing axisymmetric shock reflection is a curve with unknown shape. Therefore, to study the characteristics of the Mach reflection, one of the preconditions is to know the shape of the incident shock waves. A typical axisymmetric Mach reflection in steady flow is shown in Fig. 1.

As pointed out by Whitham [4], the study of shock wave in problems more than one dimension is "difficult due to the combination of two effects: the shock is adjusting to

(c) The Author(s). 2020 Open Access This article is licensed under a Creative Commons Attribution 4.0 International License, which permits use, sharing, adaptation, distribution and reproduction in any medium or format, as long as you give appropriate credit to the original author(s) and the source, provide a link to the Creative Commons licence, and indicate if changes were made. The images or other third party material in this article are included in the article's Creative Commons licence, unless indicated otherwise in a credit line to the material. If material is not included in the article's Creative Commons licence and your intended use is not permitted by statutory regulation or exceeds the permitted use, you will need to obtain permission directly from the copyright holder. To view a copy of this licence, visit http://creativecommons.org/licenses/by/4.0/. 


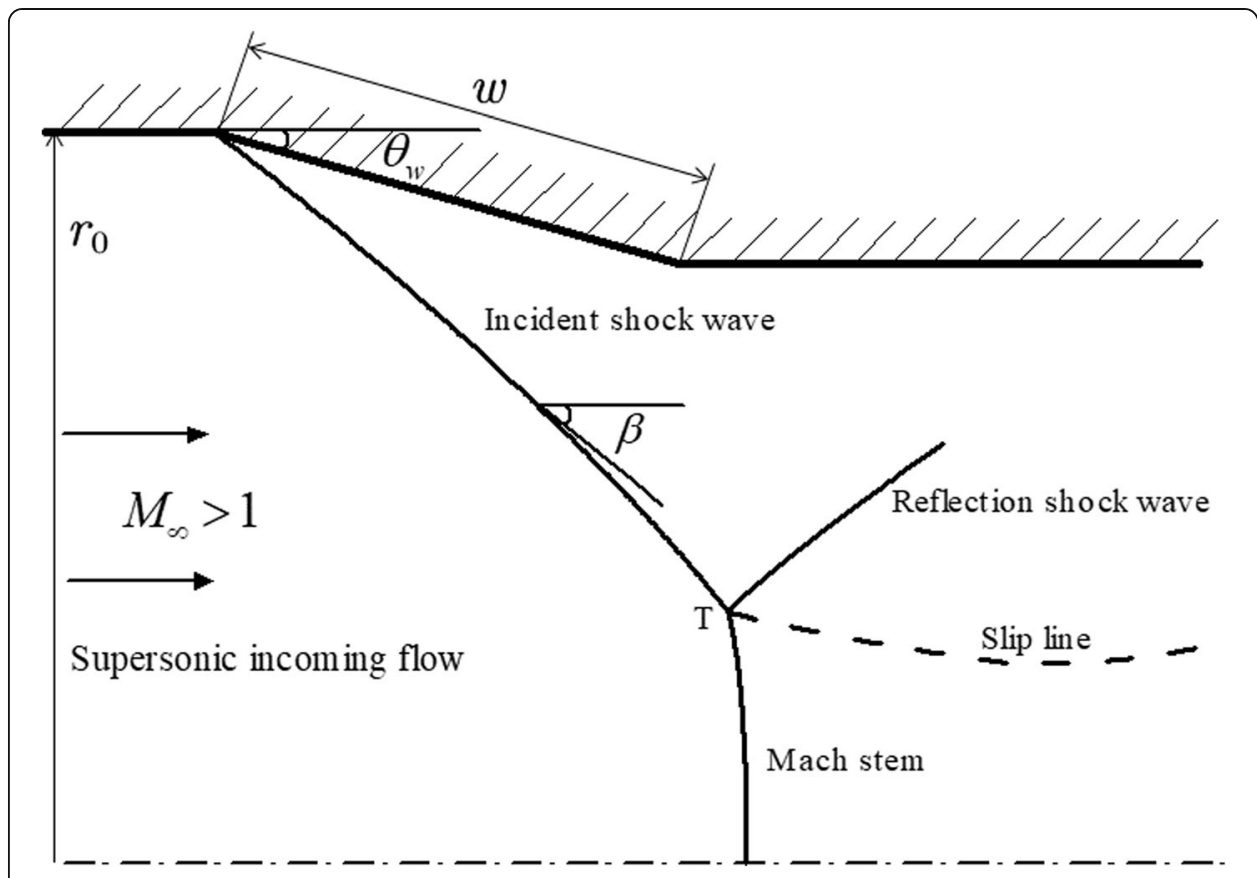

Fig. 1 Axisymmetric Mach reflection

changes in the geometry (or in the medium) at the same time that it is coping with a complicated nonlinear interaction with the flow behind it". "In the more general case, if one of the effects can be dealt with fairly simply so that emphasis can be placed on the other, there is hope for an approximate theory." The theory of geometrical shock dynamics [4] is one example of this consideration where only the geometrical effects are taken to be important. Unsteady oblique shock reflection from an axis of symmetry is studied using theory of geometrical shock dynamics by Hornung and Schwendeman [3], and the results are compared with previous numerical simulations of the phenomenon by Hornung [5]. The shock shapes, and the location of the shock-shock, are in good agreement with the numerical results. They also fit the moving incident shock shape with a generalized hyperbola based on an analogy with the Guderley singularity in cylindrical shock implosion.

However, the theory of geometrical shock dynamics is difficult to be applied in steady flow. In this paper, a simple approach to determine the shape of the incident shock wave in steady flow is proposed based on the assumption that the streamlines are straight lines at locations just behind the incident shock. The theoretical predictions of the shape of the incident shock are compared with the numerical results [6] and good agreement is observed.

\section{The shape of axisymmetric incident shock wave}

\subsection{The nonorthogonal curvilinear coordinate}

As shown in Fig. 2, at the leading edge of the conically contracting section with a half cone angle $\theta_{w}$, there is an incident shock which connects with the Mach stem and the reflected shock at the triple point. For a point on the incident shock wave, the shock 


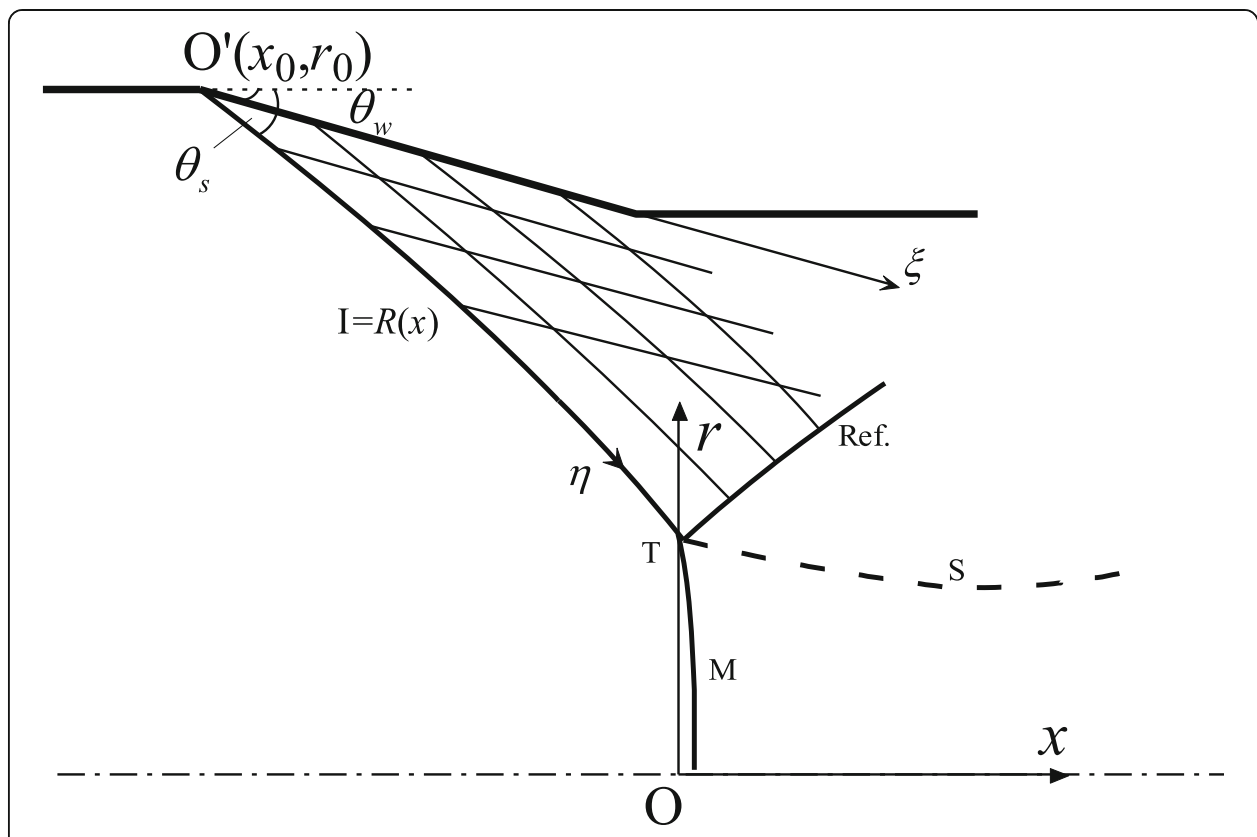

Fig. 2 Axisymmetric Mach reflection and the nonorthogonal curvilinear coordinate

angle and the deflecting angle are denoted by $\beta$ and $\theta$ respectively. The $\beta$ and $\theta$ are both positive by the definition of the present analysis.

The present study aims at finding the shape of the incident shock. To simplify the analysis, a nonorthogonal curvilinear $\xi-\eta$ coordinate is introduced as follows.

$$
\begin{gathered}
\xi=r-R(x), \\
\eta=\frac{\psi(x, r)}{V_{\infty} r_{0}} .
\end{gathered}
$$

where $r=R(x)$ is the shape of the incident shock which satisfies

$$
\frac{\mathrm{d} R}{\mathrm{~d} x}=-\tan \beta
$$

and $\psi(x, r)$ is the stream function for the axisymmetric flow, $V_{\infty}$ is the velocity of the uniform incoming flow, and $r_{0}$ is the radius of the leading edge point, $\mathrm{O}^{\prime}\left(x_{0}, r_{0}\right)$, of the conically contracting section. The physical meaning of this curvilinear coordinate is as follows. The $\xi$ coordinate with $\eta=$ constant is a family of streamlines which is used to facilitate the introduction of the basic assumption of the present paper. The $\eta$ coordinate with $\xi=$ constant is used to introduce the shape of the incident shock wave into the transformed governing equation so that a solvable equation for the shape of the incident shock wave can be derived. Indeed, $\xi=0$ is corresponding to the exact shape of the incident shock wave. We notice that the shapes of neither the incident shock wave nor the streamlines are known. However, since we are only interested in the shape of the incident shock wave, additional assumption on the streamlines at $\xi=0$ is sufficient for deriving the governing equation for the shape of the incident shock wave. Therefore, the introduction of this coordinate transform greatly simplifies the derivation of the present paper. 
In the next subsection, the steady Euler equations in $\xi-\eta$ coordinate will be derived. For this purpose, the metric terms of the transform (Eqs. (1) and (2)) will be present first. The differential relationship between two coordinates is

$$
\left(\begin{array}{l}
\mathrm{d} x \\
\mathrm{dr}
\end{array}\right)=\left(\begin{array}{ll}
x_{\xi} & x_{\eta} \\
r_{\xi} & r_{\eta}
\end{array}\right)\left(\begin{array}{l}
\mathrm{d} \xi \\
\mathrm{d} \eta
\end{array}\right)=\left(\begin{array}{ll}
\xi_{x} & \xi_{r} \\
\eta_{x} & \eta_{r}
\end{array}\right)^{-1}\left(\begin{array}{c}
\mathrm{d} \xi \\
\mathrm{d} \eta
\end{array}\right) .
$$

According to Eqs. (1) and (2), we have

$$
\left(\begin{array}{ll}
\xi_{x} & \xi_{r} \\
\eta_{x} & \eta_{r}
\end{array}\right)=\left(\begin{array}{cc}
\tan \beta & 1 \\
-\frac{r V_{r}}{r_{0} V_{\infty}} & \frac{r V_{x}}{r_{0} V_{\infty}}
\end{array}\right)
$$

where the second line of Eq. (5) is obtained following the definition of the stream function and $V_{\mathrm{r}}$ and $V_{\mathrm{x}}$ are the two components of the velocity. By the fact

$$
\tan \theta=-\frac{V_{r}}{V_{x}}
$$

and by the introduction of the notation

$$
f=\frac{r V_{x}}{r_{0} V_{\infty}}
$$

Eq. (5) can be written as

$$
\left(\begin{array}{ll}
\xi_{x} & \xi_{r} \\
\eta_{x} & \eta_{r}
\end{array}\right)=\left(\begin{array}{cc}
\tan \beta & 1 \\
f \tan \theta & f
\end{array}\right)
$$

This leads to

$$
\left(\begin{array}{ll}
x_{\xi} & x_{\eta} \\
r_{\xi} & r_{\eta}
\end{array}\right)=\frac{1}{f(\tan \beta-\tan \theta)}\left(\begin{array}{cc}
f & -1 \\
-f \tan \theta & \tan \beta
\end{array}\right) .
$$

Thus first derivatives with respect to $x$ and $r$ can be transformed into corresponding partial derivatives with respect to $\xi$ and $\eta$ by

$$
\begin{aligned}
& \frac{\partial}{\partial x}=\frac{\partial}{\partial \xi} \tan \beta+\frac{\partial}{\partial \eta} f \tan \theta, \\
& \frac{\partial}{\partial r}=\frac{\partial}{\partial \xi}+\frac{\partial}{\partial \eta} f .
\end{aligned}
$$

\subsection{The shape of the incident shock wave}

The governing equations of the present paper are the axisymmetric steady Euler equations which can be written as

$$
\begin{aligned}
& \nabla \cdot(\rho \mathbf{V})=0 \\
& (\mathbf{V} \cdot \nabla) \mathbf{V}=-\frac{1}{\rho} \nabla p \\
& (\mathbf{V} \cdot \nabla) S=0
\end{aligned}
$$

where $\mathbf{V}=V_{x} \mathbf{e}_{x}+V_{r} \mathbf{e}_{r}$ is the vector of velocity, $p$ is the pressure and $S$ is the entropy. The two components of velocity can be expressed as 


$$
V_{x}=V \cos \theta, V_{r}=-V \sin \theta
$$

Substituting Eqs. (8), (9) into Eqs. (10) - (13), we obtain the Euler equations in $\xi-\eta$ coordinate as

$$
\begin{gathered}
\left(\begin{array}{cccc}
1 & \frac{\rho}{V} & \frac{\rho(\cot \theta+\tan \beta)}{\cot \theta(\tan \theta-\tan \beta)} & 0 \\
0 & V \sin \theta \cos \theta(\tan \theta-\tan \beta) & V^{2} \cos ^{2} \theta(\tan \theta-\tan \beta) & \frac{1}{\rho} \\
0 & -V \cos ^{2} \theta(\tan \theta-\tan \beta) & V^{2} \sin \theta \cos \theta(\tan \theta-\tan \beta) & \frac{1}{\rho} \tan \beta \\
a^{2} & 0 & -1
\end{array}\right)+\frac{\partial}{\partial \xi}\left(\begin{array}{c}
\rho \\
V \\
\theta \\
p
\end{array}\right) \\
=\left(\begin{array}{c}
-\rho f \frac{\partial \theta}{\partial \eta} \frac{(\cot \theta+\tan \theta)}{\cot \theta(\tan \theta-\tan \beta)}-\frac{\rho}{r \cot \theta(\tan \theta-\tan \beta)} \\
-\frac{1}{\rho} \frac{\partial p}{\partial \eta} f \\
-\frac{1}{\rho} \frac{\partial p}{\partial \eta} f \tan \theta \\
0
\end{array}\right)
\end{gathered}
$$

This is a system of partial differential equations and it is difficult to get the shape of the incident shock by directly solving these equations. In order to overcome this difficulty, certain assumptions about the flow field behind the incident shock must be made. During the numerical simulations of the problem considered in the present paper, we find that when a steady Mach reflection can be realized in the configuration shown in Fig. 1, the streamlines just behind the incident shock have very small curvatures and can be accurately approximated by a family of straight lines. This fact is shown in Fig. 3. According to this observation, we assume in this paper that

$$
\left.\frac{\partial \theta}{\partial \xi}\right|_{s}=0
$$

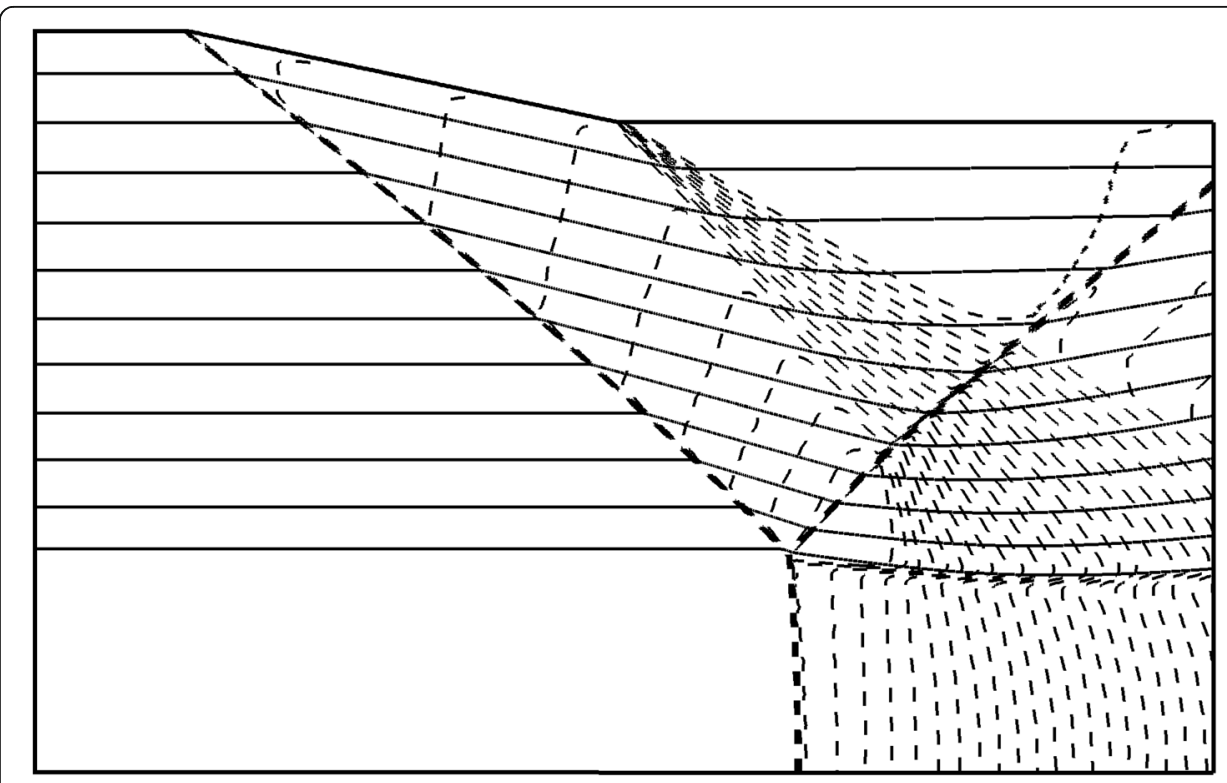

Fig. 3 The numerical results of the axisymmetric Mach reflection 
where the subscript $s$ denotes the location just behind the incident shock. Using this assumption, the problem is greatly simplified. Solving for $\frac{\partial \theta}{\partial \xi}$ using Eq. (14) yields

$$
\frac{\partial \theta}{\partial \xi}=\frac{\frac{1}{a^{2}} \frac{\partial p}{\partial \eta} f\left(\left(M^{2}-1\right)(\tan \theta-\tan \beta)\right)-\rho\left(M^{2} \sin ^{2} \theta(\cot \theta+\tan \beta)\right)\left(f \frac{\partial \theta}{\partial \eta}(\cot \theta+\tan \theta)+\frac{1}{r}\right)}{(\cot \theta+\tan \beta) \rho\left(M^{2} \sin ^{2} \theta(\cot \theta+\tan \beta)\right)-\rho M^{2}(\tan \theta-\tan \beta)^{2} \cos ^{2} \theta\left(M^{2}-1\right)} .
$$

Applying Eq. (16) and Eq. (15) at places just behind the incident shock wave, we have

$$
\left\{\left[\frac{\partial \theta}{\partial \eta}-\frac{1}{a^{2}} \frac{\partial p}{\partial \eta} \frac{(\tan \beta-\tan \theta)}{(\tan \theta \tan \beta+1)} \frac{\left(1-M^{2}\right)}{\rho M^{2}}\right](\cot \theta+\tan \theta) f+\frac{1}{r}\right\}_{s}=0 .
$$

For simplicity, we omit the subscript $s$ since it is understood that the following discussions are focused on the shape of the incident shock.

For a point on the incident shock wave, deflecting angle $\theta$ and pressure are functions of shock angle $\beta$, i.e.

$$
\tan \theta=2 \frac{\cos \beta}{\sin \beta} \frac{M_{\infty}^{2} \sin ^{2} \beta-1}{M_{\infty}^{2}(\gamma+\cos 2 \beta)+2}
$$

and

$$
\frac{p}{p_{\infty}}=1+\frac{2 \gamma}{\gamma+1}\left(M_{\infty}^{2} \sin ^{2} \beta-1\right) .
$$

In Eqs. (18) and (19), $\gamma$ is the ratio of specific heat. Therefore, Eq. (17) can be rewritten as

$$
\left[\frac{\partial \theta}{\partial \beta}-\frac{1}{a^{2}} \frac{\partial p}{\partial \beta} \frac{(\tan \beta-\tan \theta)}{(\tan \theta \tan \beta+1)} \frac{\left(1-M^{2}\right)}{\rho M^{2}}\right](\cot \theta+\tan \theta) f \frac{\partial \beta}{\partial \eta}=-\frac{1}{r} .
$$

On the incident shock curve, we have $r=R(x)$ so that

$$
\eta(x, r)=\eta(x, R(x))=\eta(x)
$$

and subsequently

$$
\left.\frac{\partial \beta}{\partial \eta}\right|_{\xi=0}=\frac{\partial \beta}{\partial x} \frac{\mathrm{d} x}{\mathrm{~d} \eta}=\frac{\partial \beta}{\partial x} \frac{-1}{f(\tan \beta-\tan \theta)}
$$

which leads to

$$
\left[\frac{\partial \theta}{\partial \beta}-\frac{1}{a^{2}} \frac{\partial p}{\partial \beta} \frac{(\tan \beta-\tan \theta)}{(\tan \theta \tan \beta+1)} \frac{\left(1-M^{2}\right)}{\rho M^{2}}\right] \frac{(\cot \theta+\tan \theta)}{(\tan \beta-\tan \theta)} \frac{\mathrm{d} \beta}{\mathrm{d} x}=\frac{1}{R} .
$$

According to Eq. (3), it is easy to derive

$$
\frac{\mathrm{d}^{2} R}{\mathrm{~d} x^{2}}=-\left(1+\tan ^{2} \beta\right) \frac{\mathrm{d} \beta}{\mathrm{d} x} .
$$

Eqs. (22) and (23) can be combined to give

$$
R_{x x}=\frac{\left(1+R_{x}^{2}\right)}{R(\cot \theta+\tan \theta)}\left[\frac{\partial \theta}{\partial \beta} \frac{1}{\left(\tan \theta+R_{x}\right)}+\frac{1}{\gamma p} \frac{\partial p}{\partial \beta} \frac{\left(1-M^{2}\right)}{M^{2}} \frac{1}{\left(1-R_{x} \tan \theta\right)}\right]^{-1} .
$$

The boundary conditions are 


$$
R_{O^{\prime}}=r_{0},\left(\frac{d R}{d x}\right)_{O^{\prime}}=\beta_{w}
$$

where $\beta_{w}$ is computed using Eq. (18) by setting $\theta=\theta_{w}$. Eq. (24) is transformed into the system of first order ordinary differential equations by the introduction of $Q=R_{x}$, which is

$$
\left\{\begin{array}{l}
R_{x}=Q \\
Q_{x}=\frac{\left(1+Q^{2}\right)}{R(\cot \theta+\tan \theta)}\left[\frac{\partial \theta}{\partial \beta} \frac{1}{\left(\tan \theta+R_{x}\right)}+\frac{1}{\gamma p} \frac{\partial p}{\partial \beta} \frac{\left(1-M^{2}\right)}{M^{2}} \frac{1}{(1-Q \tan \theta)}\right]^{-1} .
\end{array}\right.
$$

Then Eq. (25) is numerically solved using the standard four stage Runge-Kutta scheme to predict the curve of the incident shock wave. Specifically, after obtaining numerically $Q=R_{x}, \beta$ is computed using Eq. (3), $\theta, \partial \theta / \partial \beta$ and $\partial p / \partial \beta$ are derived respectively using Eqs. (18) and (19), and $M$ is updated using the oblique shock relation

$$
M^{2}=\frac{M_{\infty}^{2}+\frac{2}{\gamma-1}}{\frac{2 \gamma}{\gamma-1} M_{\infty}^{2} \sin ^{2} \beta-1}+\frac{M_{\infty}^{2} \cos ^{2} \beta}{\frac{\gamma-1}{2} M_{\infty}^{2} \sin ^{2} \beta-1} .
$$

\section{Results and discussions}

In order to validate the present analysis, the shapes of the incident shock waves predicted by solving Eq. (24) are compared with those obtained from the numerical simulations. The numerical method in the simulations is the finite volume scheme based on the rotated Riemann solver proposed by Ren (2003) [7]. The shapes of the shock waves are extracted from the numerical results using the method of Tan et al. [8].

The shapes of the incident shock waves are predicted using the present theory and the numerical simulation for several combinations of incoming flow-Mach number $M_{\infty}$ and cone half angle $\theta_{w}$ listed in Table 1 . Referring to Fig. $1, r_{0}=0.5$ is the leading edge radius, and $w$ is the length of the contracting section. The shapes of the incident shock waves are displayed in Figs. 4, 5, 6 and 7. It is seen that the theoretical curves agree very well with the simulated curves in each test cases. This indicates the hypothesis of $\frac{\partial \theta}{\partial \xi}=0$ is reasonable for the given flow conditions. It is observed in Figs. 4, 5, 6 and 7, when $r$ is large enough (close to $r_{0}$ ), the discrepancy between the numerical and theoretical curves becomes clearer, this is possibly due to the numerical viscosity of the numerical scheme, which leads to the inaccuracy in $\beta_{w}$ in the numerical results. It is also observed that when $r$ is smaller (close to the axis of symmetry), there are singularities in the theoretical predictions so that there does not exist a smooth curve of the incident shock wave all the way to the axis of symmetry. We think this phenomenon is helpful to explain the fact that there does not exist the regular shock reflection at the axis of symmetry in the axisymmetric supersonic internal flows [3].

Table 1 The flow conditions for the four additional test cases

\begin{tabular}{lllll}
\hline & Case I (Fig. 4) & Case II (Fig. 5) & Case III (Fig. 6) & Case IV (Fig. 7) \\
\hline$\theta_{w}$ & $10^{\circ}$ & $12^{\circ}$ & $12^{\circ}$ & $12^{\circ}$ \\
$M_{\infty}$ & 1.8 & 2.0 & 2.2 & 3.5 \\
$W / r_{0}$ & 0.6 & 0.6 & 0.6 & 0.6 \\
\hline
\end{tabular}




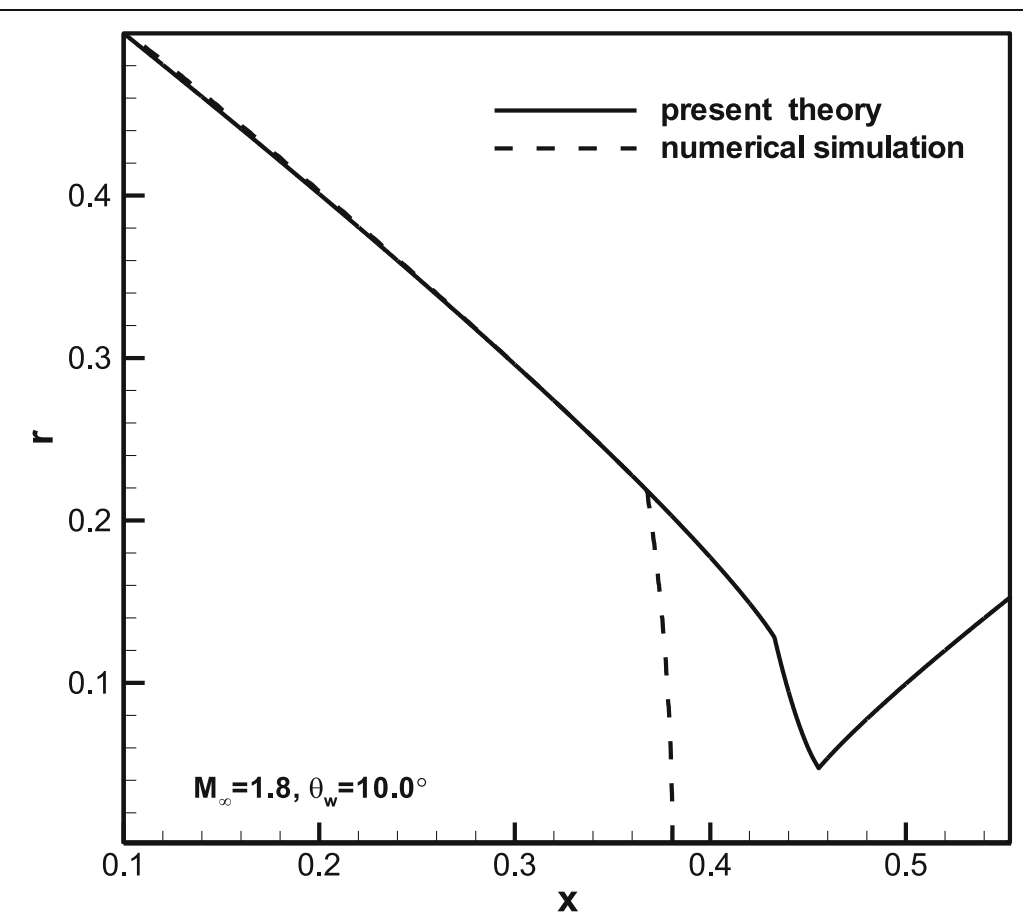

Fig. 4 The theoretical and simulated shapes of the incident shock wave when $M_{\infty}=1.8$ and $\theta_{w}=10.0^{\circ}$

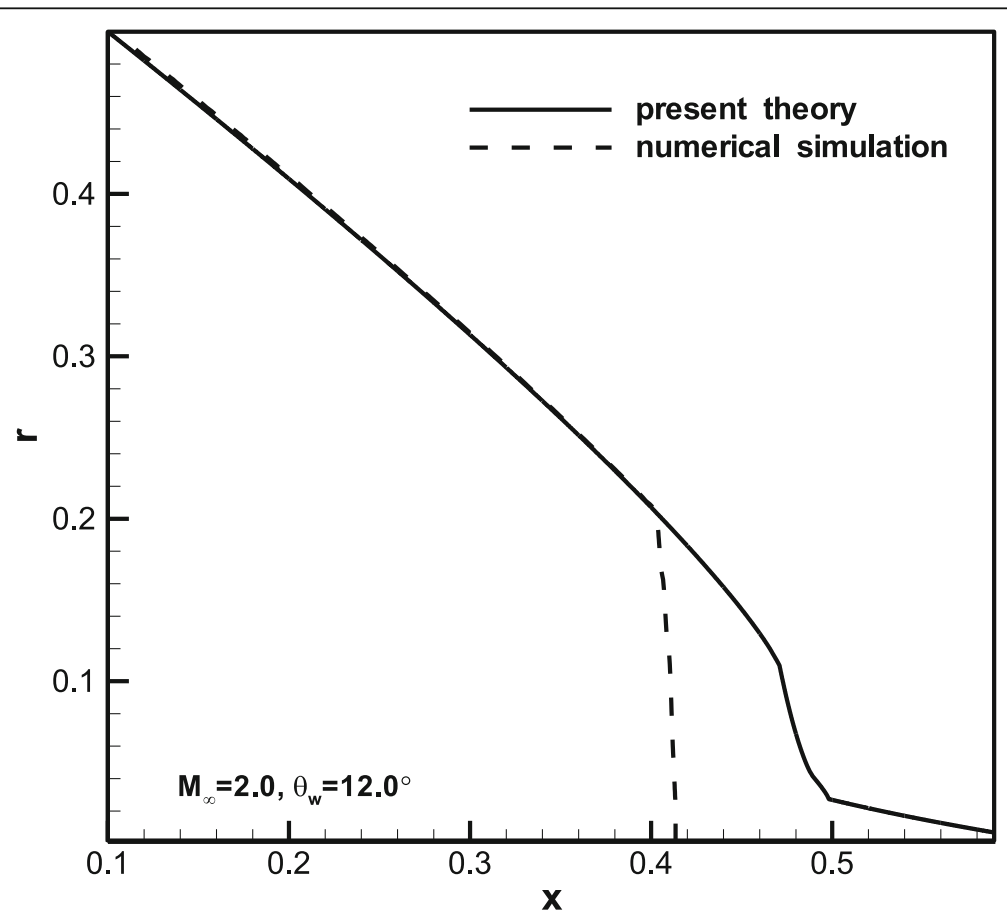

Fig. 5 The theoretical and simulated shapes of the incident shock wave when $M_{\infty}=2.0$ and $\theta_{w}=12.0^{\circ}$ 


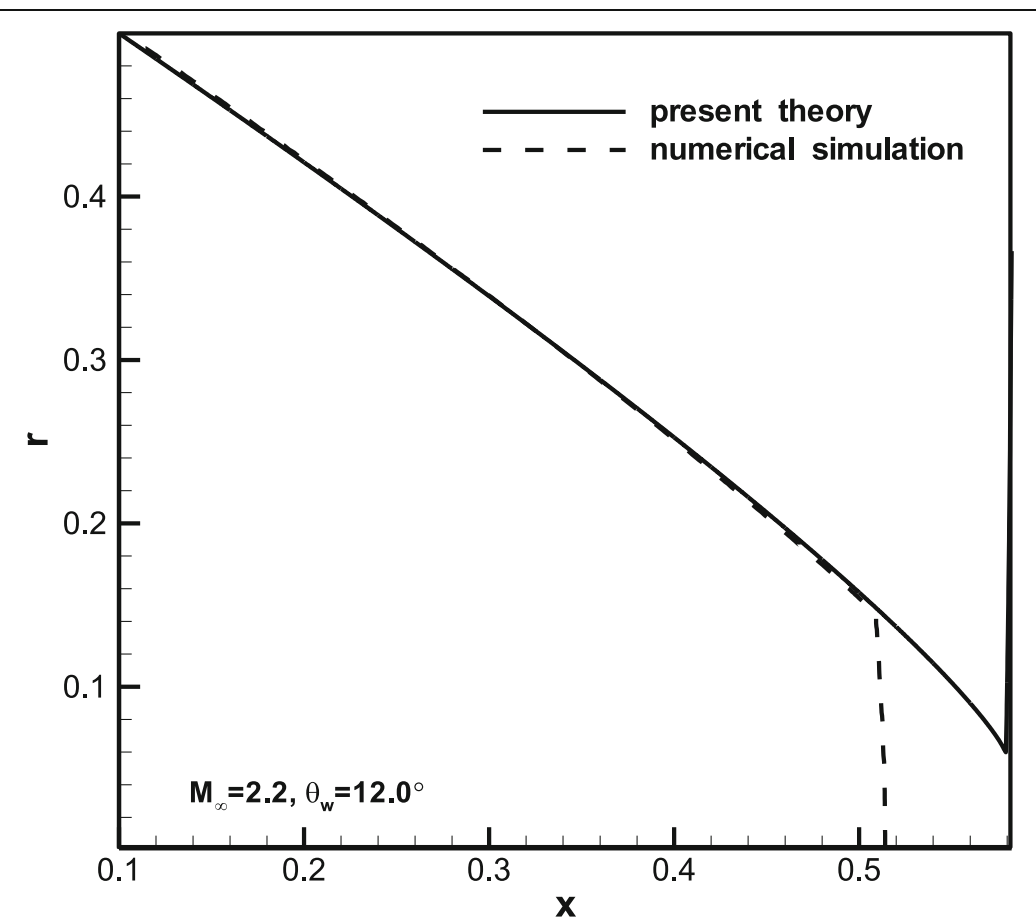

Fig. 6 The theoretical and simulated shapes of the incident shock wave when $M_{\infty}=2.2$ and $\theta_{w}=12.0^{\circ}$

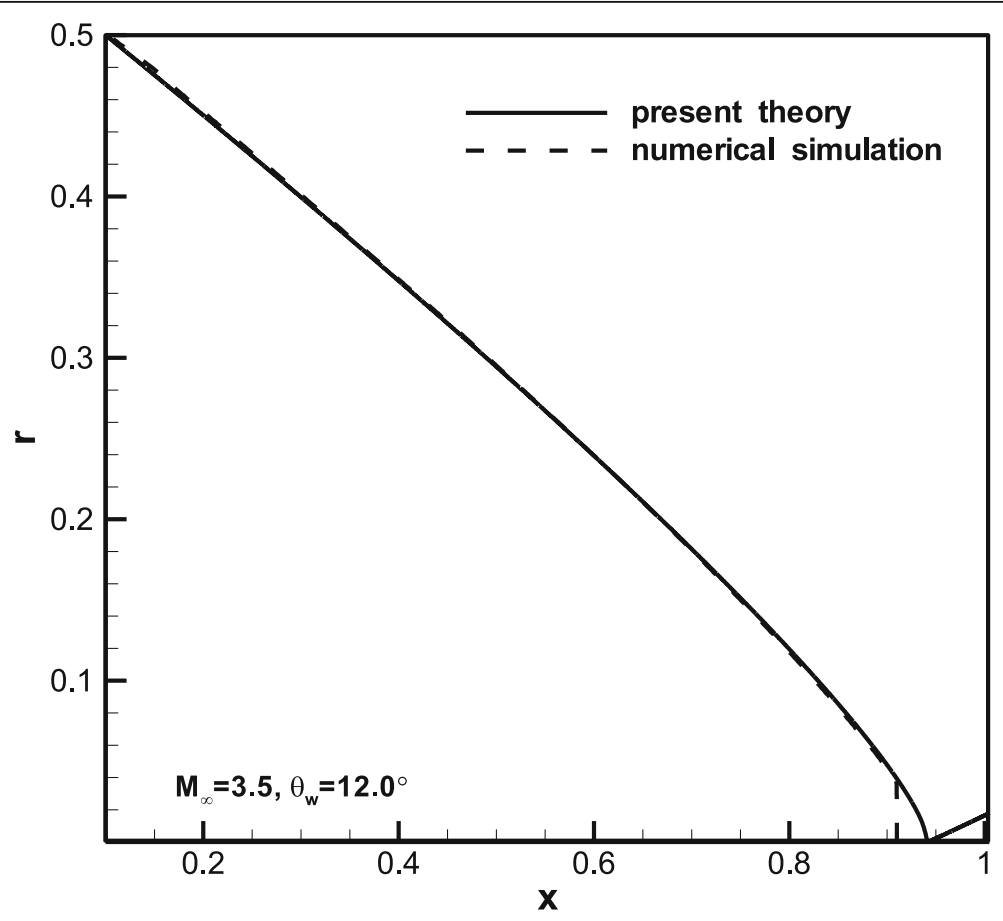

Fig. 7 The theoretical and simulated shapes of the incident shock wave when $M_{\infty}=3.5$ and $\theta_{w}=12.0^{\circ}$ 
Equation (24) indicates that the shape of the incident shock is determined by incoming flow conditions and the half cone angle $\theta_{\mathrm{w}}$. To verify this conclusion, the numerical simulations are conducted for two flows with the same incoming flow conditions and the half cone angle $\theta_{\mathrm{w}}$ but with different aspect ratios. Here the aspect ratio is defined as $w / r_{0}$, where $w$ is the diagonal length of the conically contracting section. In Fig. 8, two simulated incident shock curves are compared with the theoretical curve. The incoming-flow Mach number is 2.0 , the wedge angle is $10.0^{\circ}$, and the aspect ratios are 0.6 and 0.4 respectively. It is shown that two simulated shapes of the incident shocks are not affected by the aspect ratios and are both in good agreement with the theoretical curve.

\section{Conclusion}

In this paper, a theoretical method to predict the shape of the incident shock in steady axisymmetric Mach reflection is proposed. A nonorthogonal curvilinear $\xi-\eta$ coordinate is introduced to simplify the analysis. The basic assumption of the present paper is that the streamlines just behind the incident shock wave can be approximated by straight lines, which is strongly supported by the numerical simulations. Under this assumption, the basic flow equations are simplified to an ordinary differential equation whose solution gives the shape of the incident shock directly. The theoretical curves of the incident shock waves agree very well with the simulated ones. It is found that the shape of the incident shock wave is related only to the incoming-flow Mach number and the half cone angle.

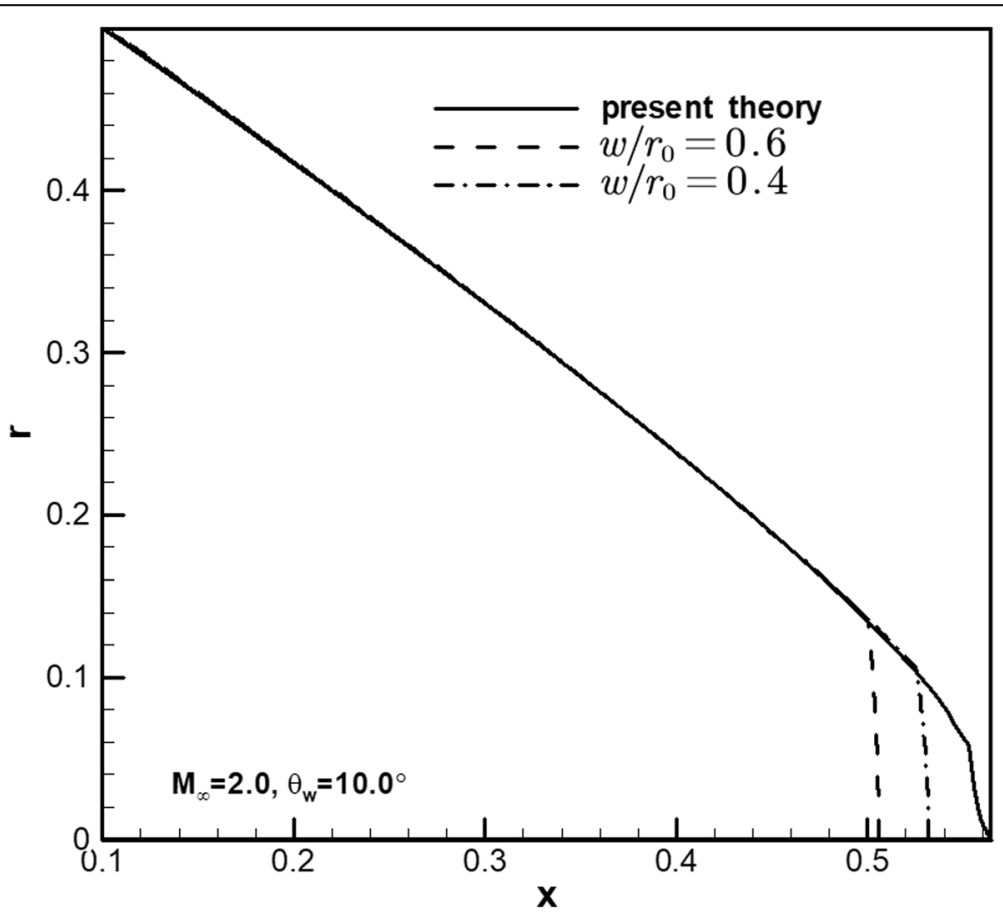

Fig. 8 The theoretical and simulated shapes of the incident shock wave when $M_{\infty}=2.0, \theta_{w}=10.0^{\circ}$. The aspect ratio is $\mathrm{w} / \mathrm{r} 0=0.6$ and $\mathrm{w} / \mathrm{r} 0=0.4$ respectively 


\section{Acknowledgements}

This work is supported by 2016YFA0401200 of national key research and development program of China and the national numerical wind tunnel project.

\section{Authors' contributions}

The methodology is proposed by the first and the third authors, the derivation of the solution is done by the first author, and the numerical simulation and comparison with theory are carried out by the second author. The author(s) read and approved the final manuscript.

\section{Funding}

This work is supported by 2016YFA0401200 of national key research and development program of China and the national numerical wind tunnel project.

\section{Availability of data and materials}

Parts of the data and materials are available upon request.

\section{Consent for publication}

This paper is submitted to AIA for the consideration of publication. The content of the manuscript has not been published, or submitted for publication elsewhere.

\section{Competing interests}

The authors declare that they have no competing interests.

\section{Author details}

${ }^{1}$ School of Aerospace Engineering, Tsinghua University, Beijing 100084, China. ${ }^{2}$ Beijing Key Laboratory of Civil Aircraft Design and Simulation Technology, Beijing Aircraft Technology Research Institute, Commercial Aircraft Corporation of China, Ltd., Beijing 102211, China.

Received: 9 July 2020 Accepted: 15 September 2020

Published online: 31 October 2020

\section{References}

1. Li H, Ben-Dor G (1997) A parametric study of Mach reflection in steady flows. J Fluid Mech 341:101-125

2. Mölder et al (1997) Focusing of conical shocks at the center-line of symmetry. In: Houwing AFP, Paull A (eds) Shock Waves. Panther Publishing and Printing, Canberra, pp 875-880

3. Hornung HG, Schwendeman DW (2001) Oblique shock reflection from an axis of symmetry: shock dynamics and relation to the Guderley singularity. J Fluid Mech 438:231-245

4. Whitham GB (1974) Linear and nonlinear waves. Wiley-Interscience, London

5. Hornung HG (2000) Oblique shock reflection from an axis of symmetry. J Fluid Mech 409:1-12

6. Ren YX et al (2005) On the Characteristics of the Mach stem. Modern Phys Lett B 19(28 \& 29):1511-1514

7. Ren YX (2003) A robust shock capturing scheme based on the rotated Riemann solver. Computers \& Fluids 32:1379-1403.

8. Tan LH, Ren YX, Wu ZN (2006) Analytical and numerical study of the near flow field and shape of the Mach stem in steady flows. J Fluid Mech 546:341-362

\section{Publisher's Note}

Springer Nature remains neutral with regard to jurisdictional claims in published maps and institutional affiliations.

\section{Submit your manuscript to a SpringerOpen ${ }^{\circ}$ journal and benefit from:}

- Convenient online submission

- Rigorous peer review

- Open access: articles freely available online

High visibility within the field

- Retaining the copyright to your article

Submit your next manuscript at $\boldsymbol{\nabla}$ springeropen.com 